\title{
Bronchogenic cyst presenting as mediastinal mass with pleural effusion
}

\author{
A. Khalil*, M.F. Carette*, B. Milleron**, M.Grivaux+, J.M. Bigot*
}

Bronchogenic cyst presenting as mediastinum mass with pleural effusion. A. Khalil, M.F. Carette, B. Milleron, M. Grivaux, J.M. Bigot. C) ERS Journals Ltd 1995.

ABSTRACT: Mediastinal bronchogenic cysts are usually identified on computed tomography (CT) as well-defined masses of variable density that may contain rim calcifications. Pleural effusion has never been described in association with these cysts.

We report two cases of bronchogenic cysts with unusual presentation because of an association with a pleural effusion not explained by pulmonary infection. The patients were studied with $\mathrm{CT}$ scan $(\mathrm{n}=2)$ and magnetic resonance imaging (MRI) of the chest $(n=1)$.

In the first case, the pleural effusion directed diagnosis towards lung tumour; and the diagnosis of bronchogenic cyst was made on thoracotomy. In the second case, bronchogenic cyst was suspected on MRI findings. Inflammatory reaction was also suspected on the CT scan, which showed enhancement of the cyst edge.

In both cases, surgical excision of the cyst was difficult because of pericystic adhesions to adjacent organs. Therefore, solely on the finding of a pleural effusion, pericystic inflammation had to be suspected.

Eur Respir J., 1995., 8, 2185-2187.

Bronchogenic cysts are congenital abnormalities resulting from embryological budding of the bronchial tree; they often arise near the carina [1-3]. Mediastinal bronchogenic cysts are frequently detected incidentally, on routine chest radiographs. They manifest as smooth and well-circumscribed masses near the carina $[2,3]$.

We describe two cases of bronchogenic cyst that appeared as mediastinal mass with pleural effusion, without pulmonary infection. To our knowledge, this radiological manifestation, in association with bronchogenic cyst, has not previously been reported.

\section{Case reports}

\section{Case 1}

A 49 year old man presented with a one month history of dysphagia. Chest radiography revealed right mediastinal mass and ipsilateral pleural effusion. Oesophagoscopy revealed a marked extrinsic compression of the oesophagus. Fibreoptic bronchoscopy showed an infiltration of the mucosa of the right intermediate bronchus. Biopsies were negative.

Chest computed tomography (CT) scans showed a mediastinal tumoral mass with tissular density (35 Hounsfield units (HU)) and a right pleural effusion (fig. la and b). CT guided needle biopsies were negative. The diagnosis of necrotic mediastinal malignancy with pleural effusion was suspected.
*Depts of Radiology and **Clinical and Intensive Care Respiratory Diseases, Tenon hospital, Paris, France. ${ }^{+}$Dept of Respiratory Diseases Meaux hospital. Meaux, France.

\author{
Correspondence: A. Khalil \\ Dept of Radiology \\ Tenon hospital \\ 4 rue de la chine \\ 75020 Paris \\ France
}

Keywords: Bronchogenic cysts

cysts

mediastinum

pleural effusion

Received: April 121995

Accepted after revision June 71995 $\mathbf{a}$

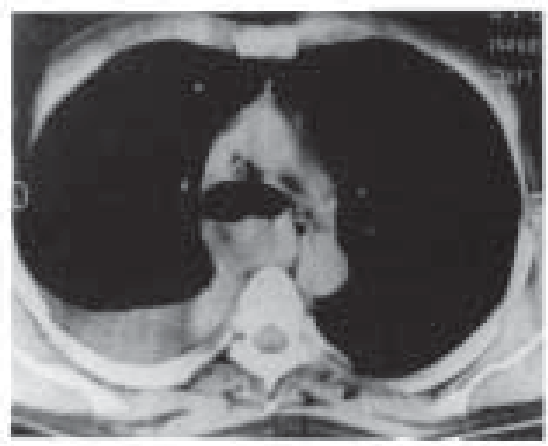

b

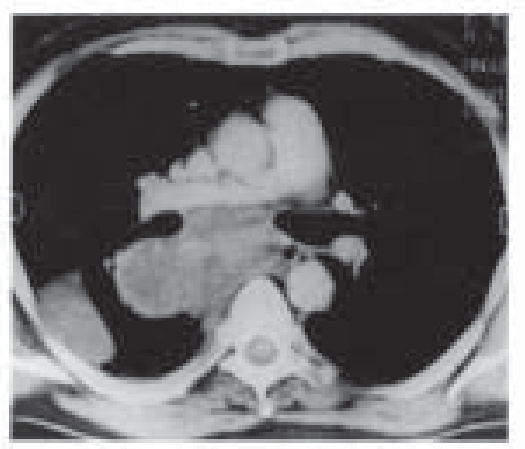

Fig. 1. - Case 1. a) Computed tomography (CT) scan $10 \mathrm{~mm}$ thick slice, medistinal windows, shows the mass behind and under the carina, associated with a right pleural effusion. b) Lower level; the mass does not enhance after contrast media injection. Enhancement of its right edge is probably related to inflammatory mediastinal pleura or to a lung collapse around the mass. Right pleural effusion is apparent. 
The patient underwent mass resection by right thoracotomy. The pleural effusion was evacuated $(400 \mathrm{~mL}$ of serosanguinous fluid), and the mediastinal mass was excised. The mass was adherent to the posterior pericardium, the right inferior pulmonary vein, and the intermediate bronchi. The oesophagus was not distinguishable from this mass. Greyish white noninfected fluid was evacuated from the cyst.

A pathological study confirmed multilocular bronchogenic cyst without neoplasm cells.

After surgery the patient progressed well and received a normal diet; he did not complain of difficulty in swallowing.

\section{Case 2}

A 58 year old woman presented with a persistent dry cough for 10 days, sometimes associated with shortness of breath. Clinical examination was normal. A chest radiograph showed a right mediastinal mass.

Fibreoptic bronchoscopy examination confirmed extrinsic narrowing of the right intermediate bronchus with calibre reduced by $50 \%$.

On unenhanced chest CT scan (fig. 2a), the mass appeared oval and had a homogeneous high density,

a

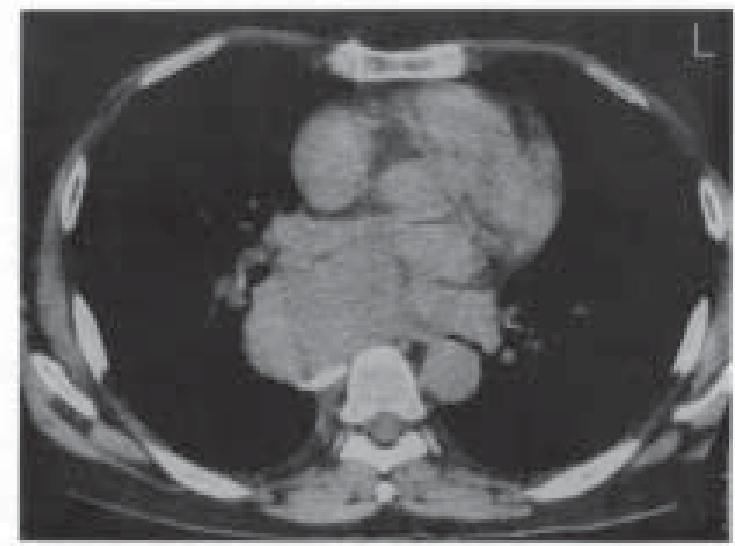

c

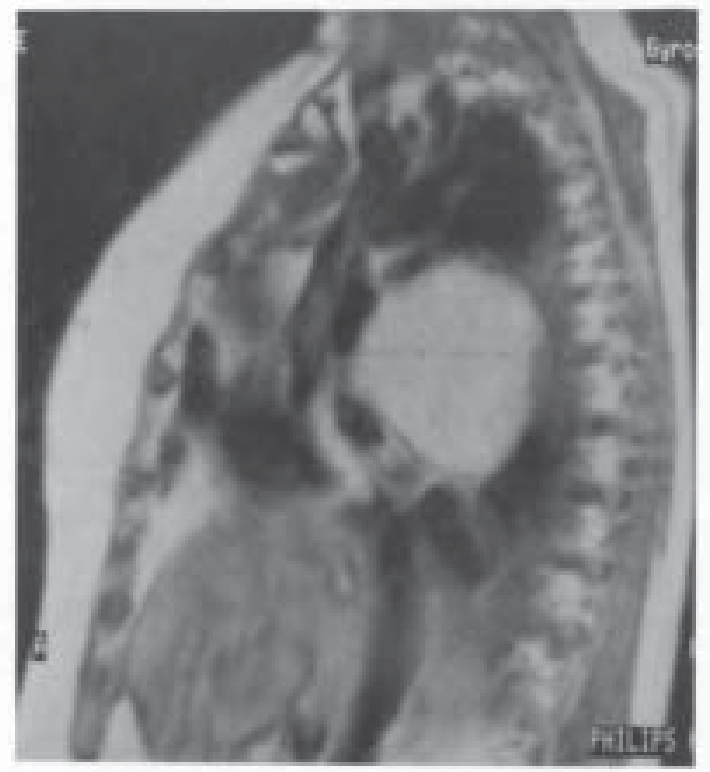

evaluated at $65 \mathrm{HU}$. It was located in the middle mediastinal compartment, in the subcarinal area; and symmetrically compressed the right intermediate bronchus. The posterior cyst wall was calcified. A small right pleural effusion was observed. After injection of contrast media (fig. 2b), the mass itself was not enhanced. Enhancement of its right edge suggested thickening of the inflammatory mediastinal pleura or a lung collapse around the mass.

Magnetic resonance imaging (MRI) demonstrated a homogeneous high signal intensity on both T1-weighted and T2-weighted sequences (fig. $2 \mathrm{c}$ and d). The pleural effusion had a low signal intensity in T1-weighted imaging and a bright signal intensity in T2-weighted imaging (Fig. 2c). The mass symmetrically compressed the right pulmonary artery.

The patient underwent mass resection by right thoracotomy. Serofibrinous pleural effusion $(800 \mathrm{~mL})$ was evacuated. Aspiration of the cyst content yielded a greyish white fluid. Bacteriological studies were negative. During dissection, the intermediate bronchus was injured, creating a small defect in the membranous wall. This defect was successfully closed without further complications. An elliptical piece of pericardium was removed because of adherence. The entire azygos arch and the right branch of vagus nerve were sacrificed.

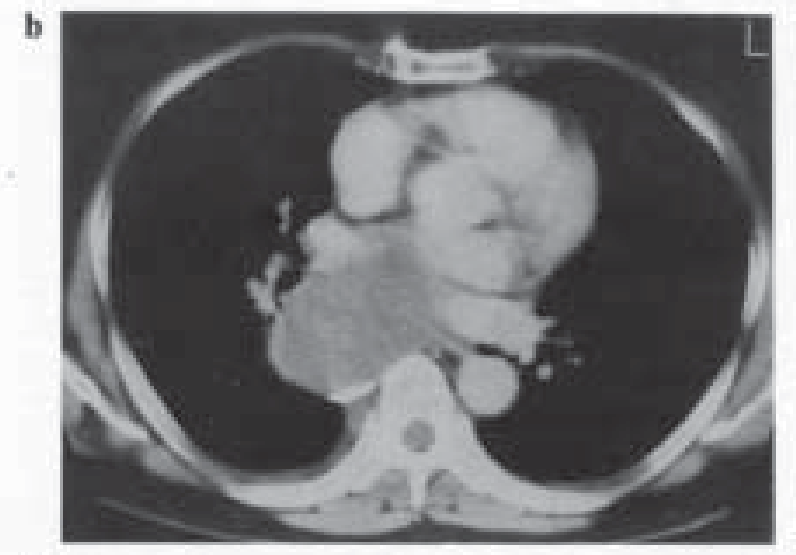

d

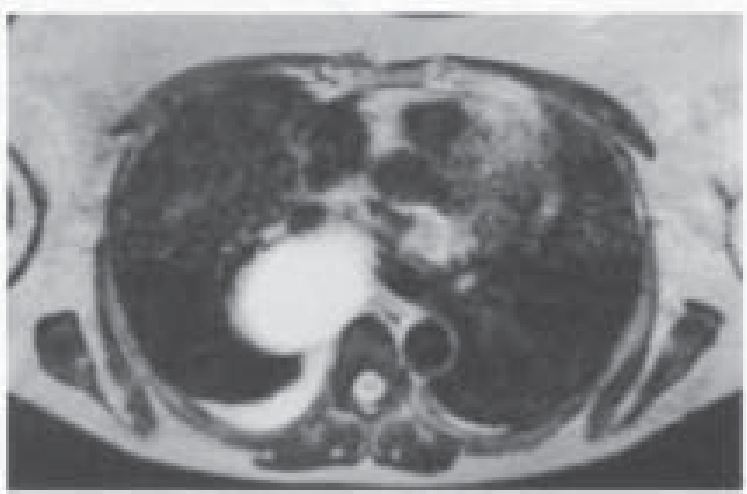

Fig. 2. - Case 2. a) Computed tomography (CT) scan without contrast media, mediastinal windows, shows an oval mass with homogeneous high density $(65 \mathrm{UH})$, peripheral calcification, and a right small pleural effusion. b) CT scan with contrast media, mediastinal windows, shows a ring enhancement which is probably related to mediastinal pleura inflammation. c) Sagittal spin echo T1-weighted image shows the medistinal mass with a high signal intensity and its anatomical relations. d) Axial spin echo T2-weighted image also shows a high signal intensity of the mass (like cerebrospinal fluid) and a pleural effusion. 
Pathological examination supported the diagnosis of bronchogenic cyst. There was found to be an inflammatory infiltration of the external cyst wall.

Three months later, the chest roentgenogram was seen to be normal.

\section{Discussion}

Bronchogenic cysts are rare cystic malformations, with walls similar to those of normal bronchi and with respiratory epithelial lining [2].

Radiographic and CT scan characteristics of bronchogenic cysts are well-defined [2-5]. On chest radiographs they are round, well-circumscribed, unilocular or multilocular masses. CT density may vary from typical water density (0-20 HU) to high density (89-99 HU), as illustrated in our cases [6]. MRI appearance is of a mass of low (grey to black) or high (white to gray) signal intensity in T1-weighted imaging, and bright (white) signal intensity in T2-weighted imaging [7, 8]. In T1 weighted images, fat, proteinaceous and haemorrhagic fluids appear in white; and water appears with a low signal intensity. In T2-weighted images water, with or without proteins, appears with a high signal intensity. In our case (Case 2), the cyst appeared with a high signal intensity in T1- and T2-weighted images; these signal abnormalities are related to an inflammatory fluid or a high protein content. CT and MRI studies are of value to clarify the relationship between the mass and the adjacent mediastinal structures before thoracotomy.

To our knowledge, pleural effusion in association with bronchogenic cyst has not previously been described. In our first case (Case 1, 1985) pleural effusion was admitted as a radiological sign against the diagnosis of bronchogenic cyst. Mediastinal mass with pleural effusion was an argument for malignancy, such as lymphoma or lung cancer. The interpretation was not the same in the second case (Case 2, 1993).

Serofibrinous or serosanguinous fluid was evacuated at surgery. These pleural effusions were probably related to an inflammatory reaction of the pleura to the bronchogenic cyst. Such inflammation could be due to the large size of the masses, perhaps with the onset of rupture. In our two cases, bronchogenic cyst excision was difficult because of dense pericystic adhesions. This inflammatory reaction was observed by means of CT scans using contrast media, as an enhancement in the cyst edge.
Percutaneous needle aspiration can be used for diagnostic purposes, or even therapeutically, if thin fluid is present within the cyst [9]. This technique for diagnosis was used in the first case and was not helpful.

Because of dense pericystic adhesions to adjacent organs, surgical excision of mediastinal bronchogenic cysts can be hazardous. A watching and waiting attitude seems inadvisable in a patient with a mediastinal cyst, even if the cyst is not symptomatic. This approach could lead to the development of complications, that tend to make operation more difficult. Other arguments for a more aggressive treatment, with total excision of these lesions, include reported cases of malignant degeneration and symptomatic recurrence [2, 3, 9].

In conclusion, the two cases presented are particularly interesting because they are the first reported cases of bronchogenic cyst with pleural effusion. Surgical treatment of these two cases was difficult. Difficulties were due to adherence to mediastinal structures, independently of the para-oesophageal characteristic of the first case. Pleural effusion could have a predictive value for these surgical difficulties.

\section{References}

1. Reed JC, Sobonya RE. Morphologic analysis of foregut cysts in the thorax. Am J Roentgenal 1974; 120: 851-860.

2. Rogers LF, Osmer JC. Bronchogenic cyst: a review of 46 cases. Am J Roentgenal 1964; 91: 273-283.

3. St-Georges R, Deslauriers J, Duranceau A, et al. Clinical spectrum of bronchogenic cysts of the mediastinum and lung in the adult. Ann Thorac Surg 1991; 52: 6-13.

4. Nakata H, Nakayama C, Kimoto $\mathrm{T}$, et al. Computed tomography of mediastinal bronchogenic cysts. J Comput Assist Tomogr 1982; 6: 733-738.

5. Mendelson DS, Rose JS, Efremidis SC, Kirschner PA, Cohen BA. Bronchogenic cysts with high CT numbers. Am J Roentgenal 1983; 140: 463-465.

6. Yernault JC, Kuhn G, Dumortier P, Rocmans P, Ketelbant P, De Vuyst P. "Solid" mediastinal bronchogenic cyst: mineralogic analysis. Am J Roentgenal 1986; 146: 73-74.

7. Nakata H, Egashira K, Watanabe H, et al. MRI of bronchogenic cysts. J Comput Assist Tomogr 1993; 17: 267-270

8. Lyon RD, McAdams HP. Mediastinal bronchogenic cyst: demonstration of a fluid-fluid level at MR imaging. Radiology 1993; 186: 427-428.

9. Suen HC, Mathisen DJ, Grillo HC, et al. Surgical management and radiological characteristics of bronchogenic cysts. Ann Thorac Surg 1993; 55: 476-481. 